\title{
The Role of Ultrasound Imaging in the Definition of the Stage of Liver Fibrosis in Patients with Chronic Hepatitis C
}

\author{
Dmitry Konstantinov, MD*; Larisa Popova, MD, ScD; Elena Konstantinova, MD, PhD; \\ Anastasia Novikova, PGS
}

Samara State Medical University, Samara, Russian Federation

\begin{abstract}
The aim of this research was to develop a method for noninvasive staging of liver fibrosis (LF) in patients with chronic hepatitis $\mathrm{C}$ (CHC) based on ultrasound imaging (UI) of the abdominal cavity.

We examined 124 patients with CHC. The diagnosis was verified on the basis of clinical and epidemiological, serological and molecular biological data. Direct ultrasonic parameters of the structure and hemodynamics of liver and spleen were supplemented with estimated indicators: square of the expected cross-section of the lobes of the liver and spleen, as well as their ratio. On the basis of the discriminant analysis of the survey data of 82 patients, we developed an analytical model (with predictive value of 95.2\%) for interval estimation of the fibrosis degree in CHC patients. We have concluded that UI performed on modern equipment, including Doppler, is able to determine the degree of LF without resorting to histological verification.
\end{abstract}

Keywords: chronic hepatitis C, ultrasound, fibrosis.

\section{Introduction}

Liver fibrosis (LF) is the main pathogenetic mechanism of liver damage in $\mathrm{HCV}$ infection, and its progression leads to liver dysfunction, development of complications, and the patient's death [1]. Evaluation of fibrosis is important for the decision to start treatment, predict the disease, and monitor the development of the cirrhosis complications [2]. Currently, none of the known methods for determining the stage of fibrosis can be considered optimal. Despite the extremely high value of histological determination of the degree of LF by using needle biopsy, this method has a number of drawbacks: the invasive nature of the procedure with the potential risk of complications and complexity of monitoring [3,4]. Noninvasive tests (transient elastography and the serum marker FibroTest), all widespread in recent years, are expensive and not included in the national standard of examination; furthermore, their specificity is insufficiently known [5]. However, the improvement of ultrasound diagnosis, the development of computer technology with improving resolution of scanners,

*Corresponding author: Dmitry Konstantinov, MD; Samara State Medical University. Samara, Russian Federation. E-mail: dk.samgmu@mail.ru and the emergence of new modes of scanning and data processing software for echo sounders enhance the diagnostic capabilities of UI [5-7].

The aim of this research was to develop a method for noninvasive staging of liver fibrosis (LF) in patients with chronic hepatitis $\mathrm{C}$ (CHC) based on ultrasound imaging (UI) of the abdominal cavity.

\section{Material and Methods}

The group inpatient and outpatient patients with $\mathrm{CHC}$ at different stages of the process were examined among patients of the Hepatological Regional Center of Samara (Samara State Medical University) during 2005-2012. The diagnosis was verified according to clinical, epidemiological, biochemical, serological and molecular-biology data. All patients underwent UI of the abdominal cavity and, in the absence of contraindications and with patient consent, hepatic biopsy. In some cases, to identify the degree of portal hypertension and the nature of co-morbidity, a fibrogastroduodenoscopy was made. We used the Toshiba 370A Power vision 6000 ultrasound scanner and two multi-frequency probes (3.0-6.0 $\mathrm{MHz}$ convex and linear 6.0-11.0 MHz). Investigation of the parameters of hepatic hemodynamics was performed in triplex 
scanning mode. Insonation angle correction was optimal in all cases. A linear probe was used to further assess the state of the contour of the liver. Part of the examination was conducted by a SONOACE X-8 ultrasound scanner using Dynamic $\mathrm{MR}^{\mathrm{TM}}$ technology, which allows the basic two-dimensional image to be represented in real-time with optimal contrast and image sharpness, maximizes the visualization of contours and boundaries of the tissues with different acoustic density at a frequency of 2.5 to $5.0 \mathrm{MHz}$.

In UI, sound conduction, echogenicity of the liver and periportal pattern were evaluated by the rank scale $(0-$ no change, 1 - reinforced, 2 - reduced); echogenicity of the liver ( 0 - no change, 1 - enhanced 2 - reduced) and periportal pattern (no change - 0 , deformed - 1 ; stress in the gate of the liver - 2, on the periphery - 3, on all fields - 4). Sixteen quantitative indicators were also recorded: obliquely-vertical size of the right lobe of the liver, $\mathrm{cm}$; the thickness of the right hepatic lobe, $\mathrm{cm}$; cross-sectional area of the right hepatic lobe $\mathrm{cm}^{2}$; cranio-caudal size of the left lobe, $\mathrm{cm}$; the thickness of the left hepatic lobe, cm; cross-sectional area of the left hepatic lobe, $\mathrm{cm}^{2}$; spleen length, $\mathrm{mm}$; spleen thickness $\mathrm{mm}$; cross-sectional area of the spleen, $\mathrm{mm}^{2}$; portal vein diameter, $\mathrm{mm}$; splenic vein diameter, $\mathrm{mm}$ (at the gate); mesenteric vein diameter, $\mathrm{mm}$; blood flow rate in portal vein $(\mathrm{cm} / \mathrm{sec})$; sectional area ratio of the liver and spleen; cross-section of the right hepatic lobe to the spleen; cross-section of the left hepatic lobe to the spleen.

Histological examination of biopsy samples (obtained by liver biopsy) was carried out after fixation in $10 \%$ buffered formalin and stained with hematoxylin and eosin. A biopsy not less than $15 \mathrm{~mm}$ and containing at least 3-4 portal tracts was considered as informative. Degree of fibrosis on the basis of histological examination of liver tissue was determined by METAVIR classification.

The data obtained were subjected to linear discriminant analysis using a stepwise algorithm and excluding discriminating variables (all ultrasonic indicators), as well as gender and age of patients. The purpose of the this analysis was to build classification functions, allowing each new observation to be referred to one of the classes, reflecting some degree of LF, according to an optimal set of discriminating variables. In accordance with the specified algorithm, in the first stage of analysis, all variables were included in the discriminant model (DM), and then at each step, variables, having a small contribution to the task of fibrosis diagnosis, were eliminated. As a determining factor for the inclusion or exclusion of variables from the model, values of the corresponding F-statistics were used. In addition to the F-statistics of each variable, at each step of the algorithm such indicators as $\lambda$ of DM as a whole, $\lambda$ of each variable of $\mathrm{DM}$, $\lambda$ private statistics of each variable of the model, F-statistics of DM as a whole, and tolerance of each variable of DM were evaluated. Mathematical and statistical analysis of data was performed using the STATISTISA 6.0.

A total of $124 \mathrm{CHC}$ patients were examined according to the specified program. Data of 82 patients were used to build $\mathrm{DM}$, and data of the remaining 42 patients were used to test the accuracy of DM.

\section{Result and Discussion}

The analysis showed that the most appropriate in terms of determining the degree of LF is DM, which includes the following discriminating variables: gender; length, width and cross-sectional area of the spleen, liver echogenicity and sound conduction, the diameter of the portal vein, the nature of periportal pattern, and the average velocity of portal blood flow. The model as a whole was statistically significant $(\lambda=0.887 ; \mathrm{F}=9.819, \mathrm{P}<0.00001)$. Other quality evaluation of DM is shown in Table 1.

\section{Table 1.}

Variables of the DM for noninvasive determination of the LF degree in patients with $\mathrm{CHC}$

\begin{tabular}{|c|c|c|c|c|c|}
\hline $\begin{array}{l}\text { Discriminating } \\
\text { variable }\end{array}$ & Wilks' $\lambda$ & Partial $\lambda$ & $F$ & $p$ & Tolerance \\
\hline Gender & 0.104 & 0.852 & 4.068 & 0.010 & 0.665 \\
\hline Spleen length & 0.111 & 0.800 & 5.818 & 0.001 & 0.017 \\
\hline Spleen width & 0.114 & 0.781 & 6.556 & 0.001 & 0.012 \\
\hline $\begin{array}{l}\text { Cross section of } \\
\text { the spleen }\end{array}$ & 0.113 & 0.784 & 6.420 & 0.001 & 0.005 \\
\hline $\begin{array}{l}\text { Sound conduction } \\
\text { of the liver }\end{array}$ & 0.107 & 0.827 & 4.894 & 0.004 & 0.601 \\
\hline $\begin{array}{l}\text { Echogenicity of } \\
\text { the liver }\end{array}$ & 0.100 & 0.887 & 2.961 & 0.038 & 0.687 \\
\hline $\begin{array}{l}\text { Portal vein } \\
\text { diameter }\end{array}$ & 0.127 & 0.698 & 10.083 & 0.000 & 0.467 \\
\hline Periportal pattern & 0.149 & 0.595 & 15.909 & 0.000 & 0.261 \\
\hline $\begin{array}{l}\text { Average speed of } \\
\text { portal blood flow }\end{array}$ & 0.178 & 0.499 & 23.412 & 0.000 & 0.517 \\
\hline
\end{tabular}

The developed DM contained the four functions of classification of fibrosis stages:

$$
\begin{gathered}
K F_{0}=-5272.10+51.77 x_{1}+103.79 x_{2}+255.72 x_{3}-2.33 x_{4}- \\
-73.36 x_{5}-37.83 x_{6}-35.79 x_{7}-59.89 x_{8}-8.37 x_{9} ; \\
K F_{1}=-5430.39+56.27 x_{1}+105.43 x_{2}+259.97 x_{3}-2.37 x_{4}- \\
-74.73 x_{5}-37.71 x_{6}-36.13 x_{7}-64.64 x_{8}-9.65 x_{9} ; \\
K F_{2}=-5381.91+55.44 x_{1}+104.72 x_{2}+258.66 x_{3}-2.35 x_{4}- \\
\quad-71.78 x_{5}-34.36 x_{6}-33.63 x_{7}-69.12 x_{8}-10.34 x_{9} ; \\
K F_{3}=-5365.65+56.55 x_{1}+104.50 x_{2}+257.58 x_{3}-2.34 x_{4}- \\
-73.28 x_{5}-33.20 x_{6}-33.42 x_{7}-70.44 x_{8}-10.68 x_{9},
\end{gathered}
$$

where $\mathrm{KF}_{0}$ - zero value of the classification stage of fibrosis; $\mathrm{KF}_{1}$ - value of the first classification stage of fibrosis; $\mathrm{KF}_{2}$ value of the second classification stage of fibrosis; $\mathrm{KF}_{3}$ - value of the third classification stage of fibrosis; $\mathrm{x}_{1}$ - gender, points (pts); $x_{2}$ - the length of the spleen, mm; $x_{3}$ - the width of the spleen, $\mathrm{mm} ; \mathrm{x}_{4}-$ cross-section of the spleen, $\mathrm{mm} ; \mathrm{x}_{5}$ - liver sound conduction pts; $\mathrm{x}_{6}$ - liver echogenicity pts; $\mathrm{x}_{7}$ - portal vein diameter, $\mathrm{mm} ; \mathrm{x}_{8}$ - periportal pattern, $\mathrm{pts} ; \mathrm{x}_{9}$ - average speed of blood flow in the portal vein, $\mathrm{cm} / \mathrm{s}$.

The desired stage of LF corresponded to the index of function of classification with the highest value. Practical application of the developed method for noninvasive determination of the degree of LF in CHC patients is shown in the following clinical examples. 
Example 1. In male patients with $\mathrm{CHC}$ the following parameters of the abdominal cavity UI were identified: spleen length - $98 \mathrm{~mm}$, the width of the spleen - $44 \mathrm{~mm}$, cross-section of the spleen $-4,312 \mathrm{~mm}$, sound conduction of the liver -0 pts, the echogenicity of the liver -2 pts, the diameter of the portal vein $-13.7 \mathrm{~mm}$, the periportal pattern $-2 \mathrm{pts}$, and the average speed of blood flow in the portal vein $-14.4 \mathrm{~cm} / \mathrm{s}$.

The values of the functions of the classification of fibrosis stages were as follows:

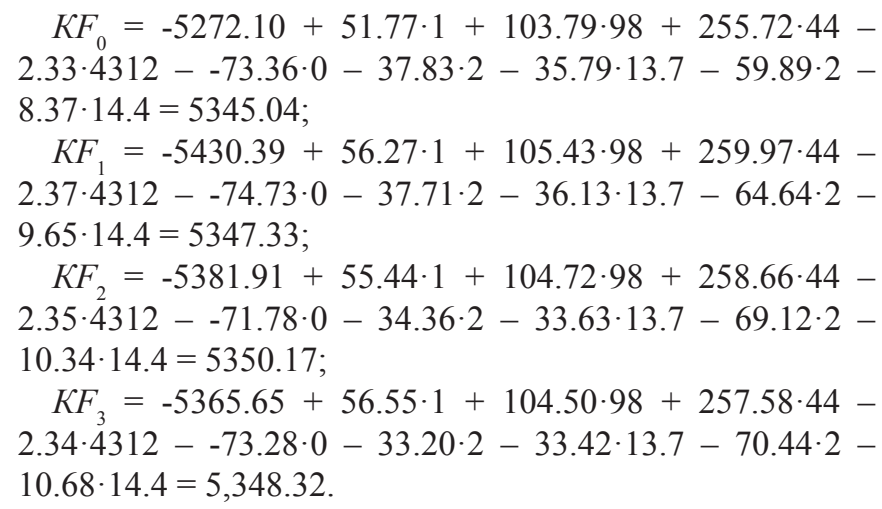

Among the calculated classification functions, the value function $\mathrm{KF} 2=5350.17$ is the highest, which corresponds to the stage of fibrosis, expressed as 2 points. In this patient, the stage of LF, according to the morphological study of liver biopsies, was also 2 points.

Example 2. In female patient with $\mathrm{CHC}$ the following parameters of the abdominal cavity UI were identified: spleen length - $99 \mathrm{~mm}$, the width of the spleen - $35 \mathrm{~mm}$, cross-section of the spleen $-3,465 \mathrm{~mm}$, sound conduction of the liver $0 \mathrm{pts}$, the echogenicity of the liver - 1 pts, the diameter of the portal vein $-12.1 \mathrm{~mm}$, periportal pattern - $0 \mathrm{pts}$, and the average speed of blood flow in the portal vein $-24.7 \mathrm{~cm} / \mathrm{s}$.

The values of the functions of the classification of fibrosis stages were as follows:

$$
K F_{0}=-5272.10+51.77 \cdot 0+103.79 \cdot 99+255.72 \cdot 35-
$$
$2.33 \cdot 3465--73.36 \cdot 0-37.83 \cdot 1-35.79 \cdot 12.1-59.89 \cdot 0-$ $8.37 \cdot 24.7=5198.66$

$K F_{1}=-5430.39+56.27 \cdot 0+105.43 \cdot 99+259.97 \cdot 35-$ $2.37 \cdot 3465--74.73 \cdot 0-37.71 \cdot 1-36.13 \cdot 12.1-64.64 \cdot 0-$ $9.65 \cdot 24.7=5187.80$

$K F_{2}=-5381.91+55.44 \cdot 0+104.72 \cdot 99+258.66 \cdot 35-$ $2.35 \cdot 3465--71.78 \cdot 0-34.36 \cdot 1-33.63 \cdot 12.1-69.12 \cdot 0-$ $10.34 \cdot 24.7=5185.27$

$K F_{3}=-5365.65+56.55 \cdot 0+104.50 \cdot 99+257.58 \cdot 35-$ $2.34 \cdot 3465--73.28 \cdot 0-33.20 \cdot 1-33.42 \cdot 12.1-70.44 \cdot 0-$ $10.68 \cdot 24.7=5179.29$.

Among the calculated classification functions, the value function $\mathrm{KF} 0=5,198.66$ is the highest, which corresponds to the stage of fibrosis, expressed as 0 points. In this patient, the stage of LF, according to the morphological study of liver biopsies, was also 0 points.

In order to determine the diagnostic significance of the obtained classifying functions, testing the accuracy of noninvasive staging of LF was carried out. To do this, in 42 CHC patients, LF was determined in two ways: test with our model, and histological examination of liver biopsy, which served as the "gold standard." The test results and the final point and interval estimates of baseline parameters of diagnostic significance are shown in Tables 2-3.

Table 2 .

Quality evaluation of DM for noninvasive determination of the $L F$ degree in patients with $\mathrm{CHC}$

\begin{tabular}{|c|c|c|c|c|c|}
\hline \multirow{2}{*}{$\begin{array}{c}\text { Noninvasive } \\
\text { evaluation }\end{array}$} & \multicolumn{4}{|c|}{ Histological stage of LF } & \multirow{2}{*}{ Total } \\
\cline { 2 - 5 } & 0 & 1 & 2 & 3 & \\
\hline 0 & $6(100 \%)$ & $10(62.5 \%)$ & $1(6.7 \%)$ & 0 & 17 \\
\hline 1 & 0 & 0 & 0 & 0 & 0 \\
\hline 2 & 0 & $5(31.3 \%)$ & $13(86.7 \%)$ & 0 & 18 \\
\hline 3 & 0 & $1(6.3 \%)$ & $1(6.7 \%)$ & $5(100 \%)$ & 7 \\
\hline Total & $6(100 \%)$ & $16(100 \%)$ & $15(100 \%)$ & $5(100 \%)$ & 42 \\
\hline
\end{tabular}

Table 3.

The point and 95\% interval estimates of baseline parameters of diagnostic significance of DM for noninvasive determination of the $L F$ degree in patients with $C H C$

\begin{tabular}{|l|c|c|c|c|}
\hline \multirow{2}{*}{$\begin{array}{c}\text { Indicator of diagnostic } \\
\text { significance }\end{array}$} & \multicolumn{4}{|c|}{ Histological stage of LF } \\
\cline { 2 - 5 } & 0 & 1 & 2 & 3 \\
\hline \multirow{3}{*}{ Sensitivity } & 0.607 & - & 0.595 & 0.549 \\
& 1.000 & 0 & 0.867 & 1.000 \\
& - & 0.171 & 0.983 & - \\
\hline \multirow{3}{*}{ Specificity } & 0.519 & 0.891 & 0.642 & 0.818 \\
& 0.694 & 1.000 & 0.815 & 0.946 \\
& 0.837 & - & 0.942 & 0.993 \\
\hline Predictive & 0.142 & - & 0.465 & 0.290 \\
value & 0.353 & - & 0.722 & 0.714 \\
& 0.617 & - & 0.903 & 0.963 \\
\hline
\end{tabular}

Table 3 shows that the point estimates of the prognostic value of diagnostics of the various stages of LF are unequal and relatively small (35.3-72.2\%). However, the analysis of residues of DM showed that the degree of fibrosis was determined accurately in $57.1 \%$ of cases. A deviation of the prognostic evaluations from the true values of LF (not more than one stage) was observed in $38.1 \%$ of the cases. Diagnostic errors equal to two stages occurred in only two cases (4.8\%): in one case, it was an overstatement of the stage; and in another case, it was a lower degree of fibrosis. The small size and symmetry of the possible deviations of the prognostic estimates with respect to zero determines how expedient the practical application of the developed method is for noninvasive staging of LF in CHC patients, provided the formulation of the final result with its possible diagnostic errors, equal to 1 stage, namely, the predictive stage of fibrosis \pm 1 stage. In interval estimation of the degree of fibrosis, the predictive value of diagnosis with $95 \%$ statistical reliability is within $83.8 \%-99.4 \%$.

Thus, ultrasound, performed on modern equipment, 
allows to determine the degree of liver fibrosis of patients with chronic hepatitis $\mathrm{C}$ without resorting to histological diagnosis with predictive value $95.2 \%$.

\section{Competing interests}

The authors declare that they have no competing interests.

\section{References}

1. Dienstag JL. The role of liver biopsy in chronic hepatitis C. Hepatology 2002; 36 (5 Suppl 1):S152-60.

2. Saadeh S, Cammell G, Carey WD, Younossi Z, Barnes $\mathrm{D}$, Easley $\mathrm{K}$. The role of liver biopsy in chronic hepatitis C. Hepatology 2001; 33(1):196-200.

3. Gebo KA, Herlong HF, Torbenson MS, Jenckes MW, Chander G, Ghanem KG, et al. Role of liver biopsy in management of chronic hepatitis $\mathrm{C}$ : a systematic review. Hepatology 2002; 36(5 Suppl 1):S161-72.

4. Seeff LB, Everson GT, Morgan TR, Curto TM, Lee WM, Ghany $\mathrm{MG}$, et al. Complication rate of percutaneous liver biopsies among persons with advanced chronic liver disease in the HALT-C trial. Clin Gastroenterol Hepatol 2010; 8(10):877-83.

5. Smith JO, Sterling RK. Systematic review: non-invasive methods of fibrosis analysis in chronic hepatitis C. Aliment Pharmacol Ther 2009; 30(6):557-76.

6. Lin ZH, Xin YN, Dong QJ, Wang Q, Jiang XJ, Zhan SH, et al. Performance of the aspartate aminotransferase-to-platelet ratio index for the staging of hepatitis C-related fibrosis: an updated meta-analysis. Hepatology 2011; 53(3):726-36.

7. Klibansky DA, Mehta SH, Curry M, Nasser I, Challies T, Afdhal NH. Transient elastography for predicting clinical outcomes in patients with chronic liver disease. J Viral Hepat 2012; 19(2):e184-93. 